\title{
CAR-dependent and CAR-independent pathways of adenovirus vector-mediated gene transfer and expression in human fibroblasts
}

\author{
Chisa Hidaka, ${ }^{1,2}$ Eric Milano, ${ }^{1}$ Philip L. Leopold, ${ }^{1}$ Jeffrey M. Bergelson, ${ }^{3}$ \\ Neil R. Hackett, ${ }^{1}$ Robert W. Finberg, ${ }^{4}$ Thomas J. Wickham, ${ }^{5}$ Imre Kovesdi, ${ }^{5}$ \\ Peter Roelvink, ${ }^{5}$ and Ronald G. Crystal ${ }^{1}$
}

${ }^{1}$ Division of Pulmonary and Critical Care Medicine, Weill Medical College of Cornell University-New York Presbyterian Hospital,
New York, New York 10021, USA
${ }^{2}$ Laboratory of Soft Tissue Research, Hospital for Special Surgery, New York, New York 10021, USA
${ }^{3}$ Children's Hospital of Philadelphia, Philadelphia, Pennsylvania 19104, USA
${ }^{4}$ Division of Infectious Diseases, Dana-Farber Cancer Institute, Boston, Massachusetts 02115, USA
${ }^{5}$ GenVec Inc., Rockville, Maryland 20852, USA

Address correspondence to: Ronald G. Crystal, Division of Pulmonary and Critical Care Medicine, Weill Medical College of Cornell University-New York Presbyterian Hospital, 520 East 70th Street, ST505, New York, New York 10021, USA. Phone: (212) 746-2258; Fax: (212) 746-8383; E-mail: geneticmedicine@mail.med.cornell.edu

Received for publication September 22, 1998, and accepted in revised form December 28, 1998.

Primary fibroblasts are not efficiently transduced by subgroup C adenovirus (Ad) vectors because they express low levels of the high-affinity Coxsackie virus and adenovirus receptor (CAR). In the present study, we have used primary human dermal fibroblasts as a model to explore strategies by which Ad vectors can be designed to enter cells deficient in CAR. Using an Ad vector expressing the human CAR cDNA (AdCAR) at high multiplicity of infection, primary fibroblasts were converted from being CAR deficient to CAR sufficient. Efficiency of subsequent gene transfer by standard Ad5-based vectors and Ad5-based vectors with alterations in penton and fiber was evaluated. Marked enhancement of binding and transgene expression by standard Ad5 vectors was achieved in CAR-sufficient fibroblasts. Expression by $\operatorname{Ad} \Delta$ RGD $\beta$ gal, an Ad5-based vector lacking the arginine-glycine-aspartate (RGD) $\alpha_{V}$ integrin recognition site from its penton base, was achieved in CAR-sufficient, but not CAR-deficient, cells. Fiber-altered Ad5-based vectors, including $(a) \operatorname{AdF}(\mathrm{pK} 7)$ Bgal (bearing seven lysines on the end of fiber) (b) $\operatorname{AdF}(\mathrm{RGD})$ Bgal (bearing a high-affinity RGD sequence on the end of fiber), and (c) AdF9sK $\beta$ gal (bearing a short fiber and Ad9 knob), demonstrated enhanced gene transfer in CAR-deficient fibroblasts, with no further enhancement in CAR-sufficient fibroblasts. Together, these observations demonstrate that CAR deficiency on Ad targets can be circumvented either by supplying CAR or by modifying the Ad fiber to bind to other cell-surface receptors.

J. Clin. Invest. 103:579-587 (1999).

\section{Introduction}

Subgroup C, replication-deficient recombinant adenoviruses (Ad) are used as vectors for gene transfer based on their ability to transduce many cell types efficiently (1-3). There is increasing evidence, however, that the efficiency of Ad vectors can be limited by a deficiency of the appropriate binding/entry mechanisms on the target cell (4-7). The binding/entry of subgroup C Ad is mediated through two specific cell-surface receptors. The knob of the Ad fiber first binds to the "Coxsackie virus and adenovirus receptor" (CAR), a 46-kDa transmembrane protein that functions as the high-affinity receptor for both subgroup C Ad and the Coxsackie B viruses (8-10). HLA class I molecules may also participate in Ad fiber binding to the cell surface (11). Ad internalization requires an interaction of an arginine-glycine-aspartate (RGD) sequence on the Ad penton base with $\alpha_{v} \beta_{3}$ or $\alpha_{v} \beta_{5}$ integrins on the cell surface $(4,6,12-19)$.

Mesenchymal cells, such as primary human fibroblasts, are one example of cells that are not efficiently infected by subgroup C Ad. Although fibroblasts express $\alpha_{V}$ integrins (20-22), they are deficient in CAR. In the present study, we have used primary human dermal fibroblasts as a model to explore strategies by which Ad vectors can be designed to enter primary human cells deficient in the high-affinity receptor but sufficient in the integrin/internalization receptor. Using an Ad vector expressing the human CAR cDNA (AdCAR) at high multiplicity of infection (moi), we have converted primary fibroblasts from being CAR deficient to CAR sufficient, enabling the study of CAR-dependent and CAR-independent mechanisms of gene transfer by subgroup C Ad vectors in primary human cells.

\section{Methods}

Cell culture. Primary human dermal fibroblasts were cultured from a normal volunteer and maintained in RPMI-1640 medium. The A549 cell line (CCL185; American Type Culture Collection, Rockville, Maryland, USA) was maintained in Dulbecco's modified essential medium. Both media were 
supplemented with $10 \% \mathrm{FBS}, 50 \mathrm{U} / \mathrm{ml}$ penicillin, $50 \mu \mathrm{g} / \mathrm{ml}$ streptomycin, and $0.1 \%$ Fungizone (all components from GIBCO BRL, Gaithersburg, Maryland, USA).

Adenovirus vectors. The recombinant Ad vectors used in this study are $\mathrm{E}^{\mathrm{a}} \mathrm{a}^{-}$, partial $\mathrm{E} 1 \mathrm{~b}^{-}$, and partial $\mathrm{E} 3^{-}$, vectors based on the Ad5 genome. The expression cassette in the $\mathrm{E} 1$ position includes the cytomegalovirus (CMV) immediate/early enhancer/promoter (except $\mathrm{Ad} \Delta \mathrm{RGD} \beta \mathrm{gal}$, which uses the Rous sarcoma virus [RSV] long terminal repeat), an artificial splice signal, the transgene, and an SV40 poly $(\mathrm{A})$ /stop signal $(23,24)$. The vectors include (a) AdCAR, containing the human CAR cDNA (8); (b) Ad $\beta$ gal, with the Escherichia coli $\beta$-galactosidase ( $\beta$ gal) gene (24); (c) AdGFP, with a humanized version of the jellyfish Aequorea victoria green fluorescent protein (GFP) gene $(25,26) ;(d)$ AdNull with no transgene (24); (e) Ad5f7CAT, a chimeric Ad5-based vector bearing the Ad7a fiber, and the chloramphenicol acetyl transferase (CAT) gene (27); (f) $\mathrm{Ad} \Delta \mathrm{RGD} \beta$ gal, with a deletion of the RGD sequence in the penton base; (g) AdF(pK7)ßgal, with seven lysine residues at the $\mathrm{COOH}$-terminal end of each fiber protein $(28,29)$; (b) $\mathrm{AdF}(\mathrm{RGD}) \beta$ gal, with a high-affinity RGD sequence GCDCRGDCFCA at the $\mathrm{COOH}$-terminal end of each fiber protein (29); and (i) AdF9sK $\beta$ gal, with a shortened fiber, including the tail and first eight shaft repeats of Ad5 fiber attached to the last Ad9 fiber shaft repeat and $\operatorname{Ad} 9 \operatorname{knob}(30,31)$. For binding studies, AdGFP was labeled with carbocyanine dye, Cy3 (Amersham Life Sciences Inc., Arlington Heights, Illinois, USA) as described previously (32). The vectors were used on the basis of physical particle concentration and were propagated, purified, and stored at $-70^{\circ} \mathrm{C}$ as described previously (33-35).

Assessment of transgene expression. All reporter gene assays were performed $24 \mathrm{~h}$ after infection with $\beta$ gal, CAT, or GFP encoding vectors. The $\beta$ gal reporter gene was assessed in cell lysates in a luminometer using the Galacto-Light kit (Tropix Inc., Bedford, Massachusetts, USA) and adjusted for total protein using the BCA assay (Bio-Rad Laboratories Inc., Hercules, California, USA). The limit of detection was $10^{3}$ relative light units/mg protein. CAT activity of cell lysates was quantified in a liquid scintillation counter as described by Neumann et al. (36). The limit of detection was $10^{2} \mathrm{dpm} / \mathrm{mg}$ protein. Below the limit of detection, 10-fold differences in amount of enzyme added to a standard amount of naive cell lysate could not be differentiated by the assay. Data points labeled below this limit indicate that no activity could be detected by the assay. GFP expression was measured by counting the number of green fluorescent cells per field by fluorescence microscopy as described by Leopold et al. (32), with a minimum of 200 cells assessed for each sample.

Characterization of CAR and $\alpha_{V}$ integrins on the surface of primary buman fibroblasts. Surface expression of CAR and $\alpha_{V}$ integrins was assessed in fibroblasts or A549 cells (as a control) using indirect immunofluorescence techniques. The anti-CAR IgG1 monoclonal antibody $(\mathrm{RmcB}$; refs. 8,37$)$ and the isotypematched control antibody (H3; ref. 38), were used at 1:1,000 dilution. Primary antibodies were visualized using Texas red or FITC goat anti-mouse Ig secondary antibodies at 1:200 dilution (both from Boehringer Mannheim GmbH, Mannheim, Germany). The FITC-conjugated monoclonal mouse anti-human $\alpha_{V}$ integrin, or control antibody (both from Coulter Corp., Miami, Florida, USA), was diluted according to the

\section{Figure 1}

Evaluation of the ability of subgroup C, serotype 5 Ad vectors to transfer and express the $\beta$ gal transgene in primary human fibroblasts compared with the A549 lung epithelial cell line. $\beta$ gal activity was assessed $24 \mathrm{~h}$ after infection with $0-5 \times 10^{4} \mathrm{pu} / \mathrm{cell}$ of Ad $\beta$ gal or $5 \times 10^{4} \mathrm{pu} / \mathrm{cell}$ AdNull. The dashed line represents the limit of detection of the assay $\left(10^{3}\right.$ relative light units/mg protein). Each data point represents the mean \pm SE of triplicate measurements. Ad, adenovirus. manufacturer's recommendations and applied after labeling with RmcB and Texas red secondary antibody. Images were collected as described by Leopold et al. (32). Exposure conditions and image manipulations were identical for comparable experimental and control panels.

Modification of Ad infection of fibroblasts by prior infection with AdCAR. To evaluate Ad binding, fibroblasts were infected with AdCAR, AdNull (both $10^{4}$ particle units [pu]/cell), or no virus, and $24 \mathrm{~h}$ later incubated with $10^{11} \mathrm{pu} / \mathrm{ml}$ of Cy3-labeled AdGFP for $10 \mathrm{~min}$ at $37^{\circ} \mathrm{C}$. A FITC secondary antibody was used to visualize CAR in the green channel, whereas Cy3-AdGFP was visualized in the red channel. Images were collected in fields that included both CAR-positive and CAR-negative cells and triplicate gray-scale measurements made in arbitrary regions within the background (no cells) and within each cell in both green and red channels. Green and red fluorescence intensities were expressed as a ratio of cell to background fluorescence, and the correlation was calculated. Thirty-eight cells were assessed in this manner.

To quantify the efficiency of gene transfer by first generation Ad vectors in a population of cells that were either CAR-deficient or CAR-sufficient, human fibroblasts were first infected with AdCAR, AdNull (both $0-5 \times 10^{4} \mathrm{pu} / \mathrm{cell}$ ), or no virus (mock-infected), then infected a second time $24 \mathrm{~h}$ later with $300 \mathrm{pu} / \mathrm{cell}$ of Ad $\beta$ gal or $200 \mathrm{pu} / \mathrm{cell}$ of AdGFP. The AdGFP-infected fibroblasts were also labeled with $\mathrm{RcmB}$ and Texas red secondary antibody.

To demonstrate that the improved expression of transgene in CAR-sufficient fibroblasts resulted from a fiber-CAR interaction, fibroblasts infected with AdCAR, AdNull (both $10^{4}$ $\mathrm{pu} / \mathrm{cell}$ ), or no virus were incubated with increasing concentrations of purified Ad5 fiber (4) $\left(0-1 \mu \mathrm{g} / \mathrm{ml}\right.$ at $\left.37^{\circ} \mathrm{C}\right)$ for $1 \mathrm{~h}$ before, and also during, infection with $300 \mathrm{pu} / \mathrm{cell}$ Ad $\beta$ gal.

For all experiments using fiber- or penton-altered Ad vectors, fibroblasts were first infected with high moi AdCAR or AdNull (both $10^{4} \mathrm{pu} / \mathrm{cell}$ ) or no virus followed $24 \mathrm{~h}$ later by a second infection with low moi $(300 \mathrm{pu} / \mathrm{cell})$ of altered vector or its control. Altered vectors included Ad5f7CAT, for which standard Ad5CAT served as control, and Ad $\triangle$ RGD $\beta$ gal, $\operatorname{AdF}(\mathrm{pK} 7) \beta g a l, \operatorname{AdF}(\mathrm{RGD}) \beta g a l$, or AdF9sKßgal, for which standard Ad $\beta$ gal served as control.

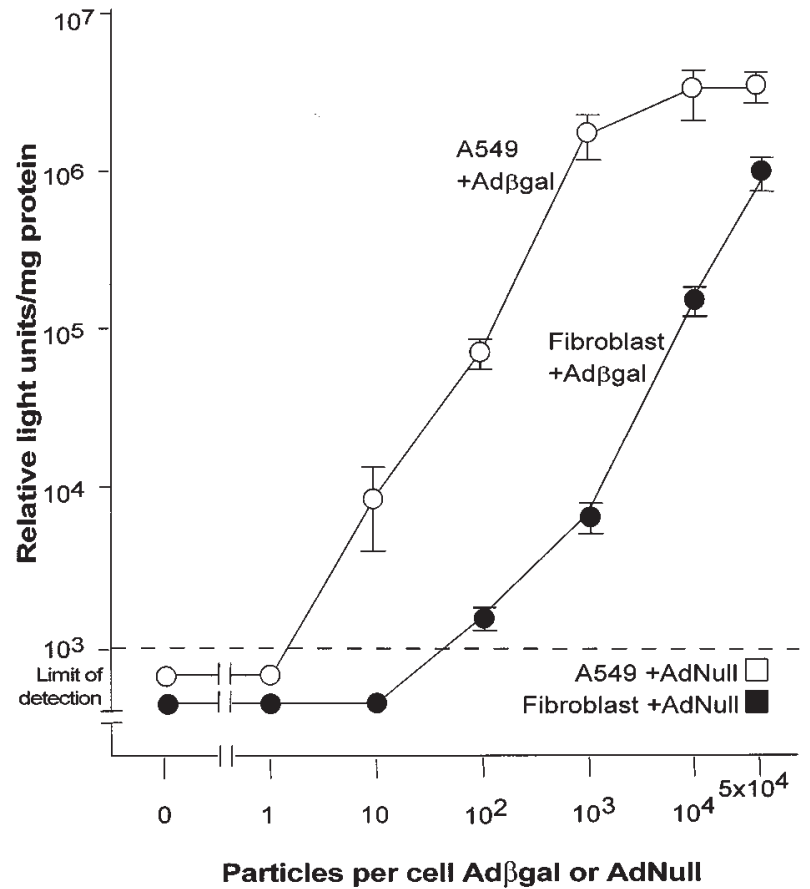


Statistical analysis. All studies were carried out in triplicate. All data are presented as mean \pm SEM. All comparisons were made using the two-tailed Student's $t$ test. For fluorescent binding studies, correlation of Ad binding and CAR expression were calculated using Spearman's rank correlation.

\section{Results}

Gene transfer and expression of first generation Ad vectors in primary buman dermal fibroblasts. A dose-response study comparing $\beta$ gal expression in human fibroblasts to A549 demonstrated that a much higher moi of Adßgal was required to generate comparable $\beta$ gal expression in the fibroblasts (Fig. 1). In fibroblasts infected with 100 $\mathrm{pu} / \mathrm{cell}$, the $\beta$ gal activity in these cells was approximately 100 -fold less than in that of A549 cells infected with the same low moi (all $P<0.01,10-5 \times 10^{4} \mathrm{pu} /$ cell; Fig. $1)$. Interestingly, the amount of $\beta$ gal expression in the A549 cells was maximal at $>10^{3} \mathrm{pu} /$ cell, whereas $\beta$ gal expression by the fibroblasts increased as a function of moi, so that with $5 \times 10^{4} \mathrm{pu} / \mathrm{cell}$, the expression of $\beta$ gal in the fibroblasts was only fourfold less than that of the A549 cells at the same moi. The control AdNull vector induced $\beta$ gal activity below the limit of detection in either A549 cells or fibroblasts.

Characterization of Ad cell-surface receptors. Primary human fibroblasts lack immunodetectable CAR (Fig. 2). A549 cells, which are easily infected with low moi of Ad (Fig. 1), demonstrated punctate cell-surface labeling when stained for CAR (Fig. $2 a$ ). In contrast, naive fibroblasts stained for human CAR demonstrated no fluorescence over background (Fig. 2c). Although the fibroblasts did not exhibit detectable CAR, they did express $\alpha_{V}$ integrins on the cell surface (Fig. $3 a$ ).

AdCAR-induced upregulation of CAR expression in fibroblasts. Fibroblasts infected with a high moi of AdCAR were made CAR-sufficient, demonstrating intense cell-surface staining of CAR as detected with an anti-CAR antibody (Fig. 2e) relative to a control antibody (Fig. 2f). In contrast, AdNull-infected fibroblasts demonstrated no cellsurface CAR (Fig. 2d). The immunofluorescent labeling in the AdCAR-infected fibroblasts was qualitatively more intense than that seen in the A549 cells. Neither infection with AdCAR nor AdNull altered the expression of $\alpha_{\mathrm{V}}$ integrin (Fig. 3, $b-d$ ).

Ad binding to CAR-deficient and CAR-sufficient fibroblasts. CAR-sufficient fibroblasts demonstrated greater binding of Cy3-AdGFP than did CAR-deficient controls (Fig. 4). Cy3-AdGFP binding to mock- and AdNull-infected cells was minimal (not shown). CAR expression among AdCAR-infected fibroblasts was not uniform. Quantitation of fluorescence intensity in AdCAR-infected cultures demonstrated that there was a positive correlation between green fluorescence (i.e., CAR expression) and red fluorescence (i.e., virus binding), with Spearman correlation analysis demonstrating a rho of 0.8 with $P<0.001$ (Fig. 5). Thus, with increasing CAR expression, the fibroblasts were able to bind more virus.

Improvement of Ad transgene expression in CAR-sufficient fibroblasts. Two strategies were used to demonstrate that AdCAR infection allows fibroblasts to be subsequently infected with an Ad vector at a low moi. First, mock-, AdNull-, or AdCAR-infected fibroblasts were infected with

\section{Table 1}

Enhanced Ad vector-mediated transfer and expression of the CAR cDNA and the green fluorescent protein gene in primary human fibroblasts resulting from prior infection with AdCAR

\begin{tabular}{lcc}
\hline & CAR-positive (\%) & GFP-positive (\%) \\
Naive fibroblasts & 0 & 0 \\
+ AdNull & 0 & $1 \pm 1 \%$ \\
+ AdCAR & $57 \pm 7 \%$ & $31 \pm 2 \%$
\end{tabular}

Primary human fibroblasts were infected with AdCAR or AdNull (both $10^{4}$ $\mathrm{pu} /$ cell) or mock-infected (naive). After $24 \mathrm{~h}$, the cells were exposed for $1 \mathrm{~h}$ to AdGFP (coding for green fluorescent protein, $200 \mathrm{pu} / \mathrm{cell}$ ). The cells were then trypsinized, plated onto coverslip dishes, and, $48 \mathrm{~h}$ later, incubated with anti-CAR antibody or isotype control followed by a Texas red secondary antibody. The percentage of green $\left(\mathrm{GFP}^{+}\right)$and red fluorescent cells $\left(\mathrm{CAR}^{+}\right)$were quantified by evaluation of 200 cells by immunofluorescent microscopy. The data are presented as the mean \pm SEM of triplicate measurements. Ad, adenovirus; $C A R$, Coxsackie virus and adenovirus receptor; GFP, green fluorescent protein.

a low moi (200 pu/cell) of AdGFP and labeled with antiCAR antibody. The number of cells expressing CAR was compared with the number of cells expressing GFP. After infection with AdCAR and then AdGFP, 57\% of fibroblasts were CAR-positive, and 31\% expressed GFP (Table 1). All GFP-positive cells in this group were also CAR positive. In contrast, fibroblasts infected with AdNull and then AdGFP expressed no detectable CAR $(P<0.001)$, and only $1 \%$ expressed GFP $(P<0.01)$. As a second approach, mock-, AdNull-, or AdCAR-infected fibroblasts were infected with a low moi (300 pu/cell) of Adßgal. Compared with CARdeficient controls, CAR-sufficient fibroblasts demonstrated higher transgene expression (Fig. 6). $\beta$ gal expression derived from a fixed, low moi infection with Ad $\beta$ gal, depended on the dose of AdCAR used as the first vector over a 3 log range. In AdNull-infected cells, there was a minor increase in the $\beta$ gal expression (a maximum of 10fold above that of no first vector). In contrast, the $\beta$ gal expression observed in AdCAR-infected fibroblasts was markedly increased (1000-fold above that of no first vector at $5 \times 10^{4} \mathrm{pu} / \mathrm{cell}, P<0.01 ; 200$-fold above that of AdNull first vector, $P<0.02)$.

Fiber-CAR interaction in the second infection of CAR-sufficient fibroblasts. Transgene expression in CAR-sufficient, but not CAR-deficient, cells was suppressed by the presence of Ad5 fiber (Fig. 7). In CAR-sufficient fibroblasts, the competitive effect of recombinant Ad5 fiber was significant, decreasing $\beta$ gal activity by up to $80 \%$ in a dose-dependent manner (Fig. 7). In contrast, the presence of recombinant Ad5 fiber only minimally affected $\beta$ gal expression in the CAR-deficient controls.

No enhancement of transgene expression in CAR-sufficient vs. CAR-deficient cells was observed when a chimeric virus that bears an Ad7a fiber rather than Ad5 fiber was used for the second infection (Fig. 8). As expected, CAT activity in CAR-sufficient cells infected with the standard Ad5-based Ad5CAT vector was 80-fold greater when compared with naive cells and 20-fold greater than AdNull-infected controls ( $P<0.01$, both comparisons). In contrast, CAT activity in CAR-sufficient cells did not differ significantly from that of AdNull-infected control $(P>0.6)$ when the second infection was with Ad5f7CAT. The relative importance of the fiber-CAR interaction versus the integrin-penton interaction was evaluated by 


\section{Figure 2}

CAR expression on primary human fibroblasts. Twenty-four hours after infection with AdCAR, AdNull (both $10^{4} \mathrm{pu} / \mathrm{cell}$ ), or no virus (naive), fibroblasts were incubated with anti-CAR antibody ( $R m c B$ ) or isotypematched control antibody followed by Texas red-labeled secondary antibody and examined by fluorescent microscopy. Naive A549 cells served as positive control. (a) A549, RmcB. (b) A549, control antibody. (c) Naive fibroblasts, RmcB. (d) AdNull-infected fibroblasts, RmcB. (e) AdCARinfected fibroblasts, RmcB. (f) AdCAR-infected fibroblasts, control antibody. Scale bar, $10 \mu \mathrm{m}$. CAR, Coxsackie virus and adenovirus receptor. comparing $\beta$ gal expression in CAR-sufficient and CARdeficient fibroblasts infected with either standard Ad $\beta$ gal or $A d \Delta R G D \beta g a l$. Although $\beta$ gal expression by $\mathrm{Ad} \Delta$ RGD $\beta$ gal in mock-infected fibroblasts was below the limit of detection, expression with the $\operatorname{Ad} \Delta \mathrm{RGD} \beta \mathrm{gal}$ vector in CAR-sufficient fibroblasts was significant, approximately 10 -fold above that of the background and fivefold greater than that of the AdNull control $(P<0.02$; Fig. 9). This suggests that upregulation of CAR can improve Ad infection even in the absence of the penton-integrin interaction.

Gene transfer and expression mediated by fiber-altered vectors. Ad vectors bearing a variety of modifications in their fiber proteins bypass the usual fiber-CAR interaction and can be used to infect CAR-deficient cells (Fig. 10). Compared with the standard Ad5-based Ad $\beta$ gal vector, fiber-altered vectors demonstrated improved transgene expression in CAR-deficient fibroblasts. AdF(pK7)ßgal was the most effective, inducing an approximately 200 fold increase in $\beta$ gal expression in naive fibroblasts versus standard Ad $\beta$ gal $(P<0.01)$. An approximately 100 fold increase was observed with AdF(RGD) $\beta$ gal $(P<0.01$ compared to standard Ad $\beta$ gal), and an approximately
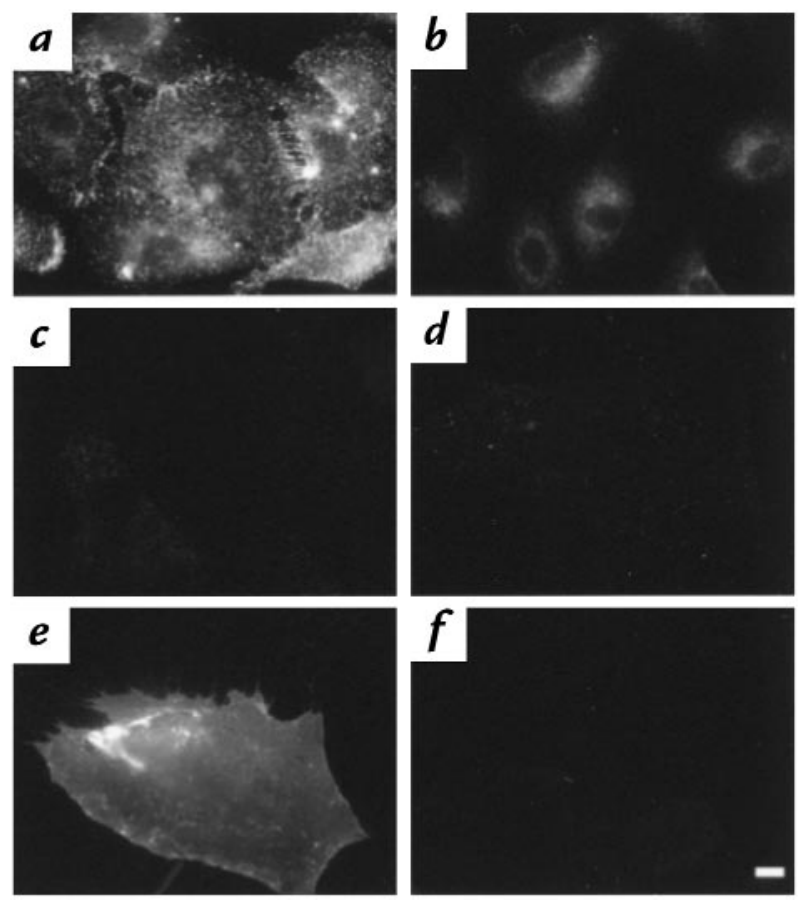

eightfold increase was observed with AdF9sK $\beta$ gal $(P<$ 0.01 compared with standard Ad $\beta$ gal). Whereas CARsufficient fibroblasts demonstrate a 70-fold increase in $\beta$ gal expression over that of AdNull-infected controls $(P$ $<0.001)$ when infected with standard Ad $\beta$ gal, no significant difference was found between CAR-sufficient versus CAR-deficient cells infected with AdF(pK7)ßgal $(P>$ $0.8)$ or AdF9sKßgal $(P>0.1)$. These data suggest that the mechanism for binding and/or entry by these vectors is CAR-independent. With AdF(RGD)ßgal, a small (about 1.5 -fold) increase in $\beta$ gal expression was noted in the AdCAR-versus the AdNull-infected cells $(P<0.03)$.

\section{Discussion}

Gene therapy by Ad-mediated gene transfer requires that the target cells bind and take up the Ad vector efficiently $(15,20-22)$. In this study, we have demonstrated that CAR is rate limiting for infection by standard Ad5 vectors in primary $\alpha_{V}$ integrin-positive cells and that the upregulation of CAR in such cells leads to increased binding of Ad, as well as more efficient expression of Ad transgene, both of which depend on a subgroup-specific fiber-CAR interaction. Using a penton base RGD-deficient Ad vec-

\section{Figure 3}

$\alpha_{v}$ integrin in AdCAR- and AdNull-infected fibroblasts. Twenty-four hours after infection with AdCAR or AdNull (both $10^{4} \mathrm{pu} / \mathrm{cell}$ ) or no vector, fibroblasts were double-labeled with RmcB and anti- $\alpha_{v}$ integrin antibody. (a) Naive fibroblasts, $\alpha_{v}$ integrin antibody. (b) AdNull-infected fibroblasts, $\alpha_{v}$ integrin antibody. (c) AdCAR-infected fibroblasts, anti- $\alpha_{v}$ integrin antibody. (d) AdCAR-infected fibroblasts, RmcB. Scale bar, $10 \mu \mathrm{m}$.
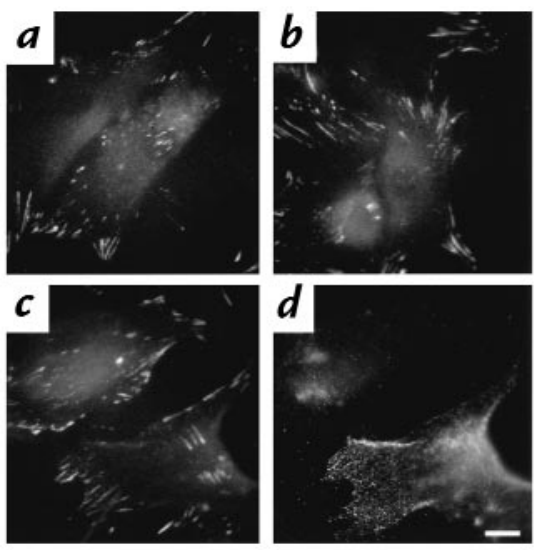


\section{Figure 4}

Binding of fluorescent Ad vectors by CAR-sufficient primary fibroblasts. AdCAR- infected fibroblasts were incubated with Cy3-labeled fluorescent AdGFP ( $\left.10^{11} \mathrm{pu} / \mathrm{cell}\right)$ for $10 \mathrm{~min}$ at $37^{\circ} \mathrm{C}$. The cells were then fixed and stained for CAR as described for Fig. 2, but with FITClabeled secondary antibody. Naive A549 cells served as positive control. (a) A549 cells, DIC. (b) A549 cells, Cy3-AdGFP binding. (c) A549 cells, RmcB. (d) AdCAR-infected fibroblasts, DIC. (e) AdCAR-infected fibroblasts, Cy3-Ad5GFP binding. ( $f$ ) AdCAR-infected fibroblasts, RmcB. Scale bar, $10 \mu \mathrm{m}$. DIC, differential interference contrast.
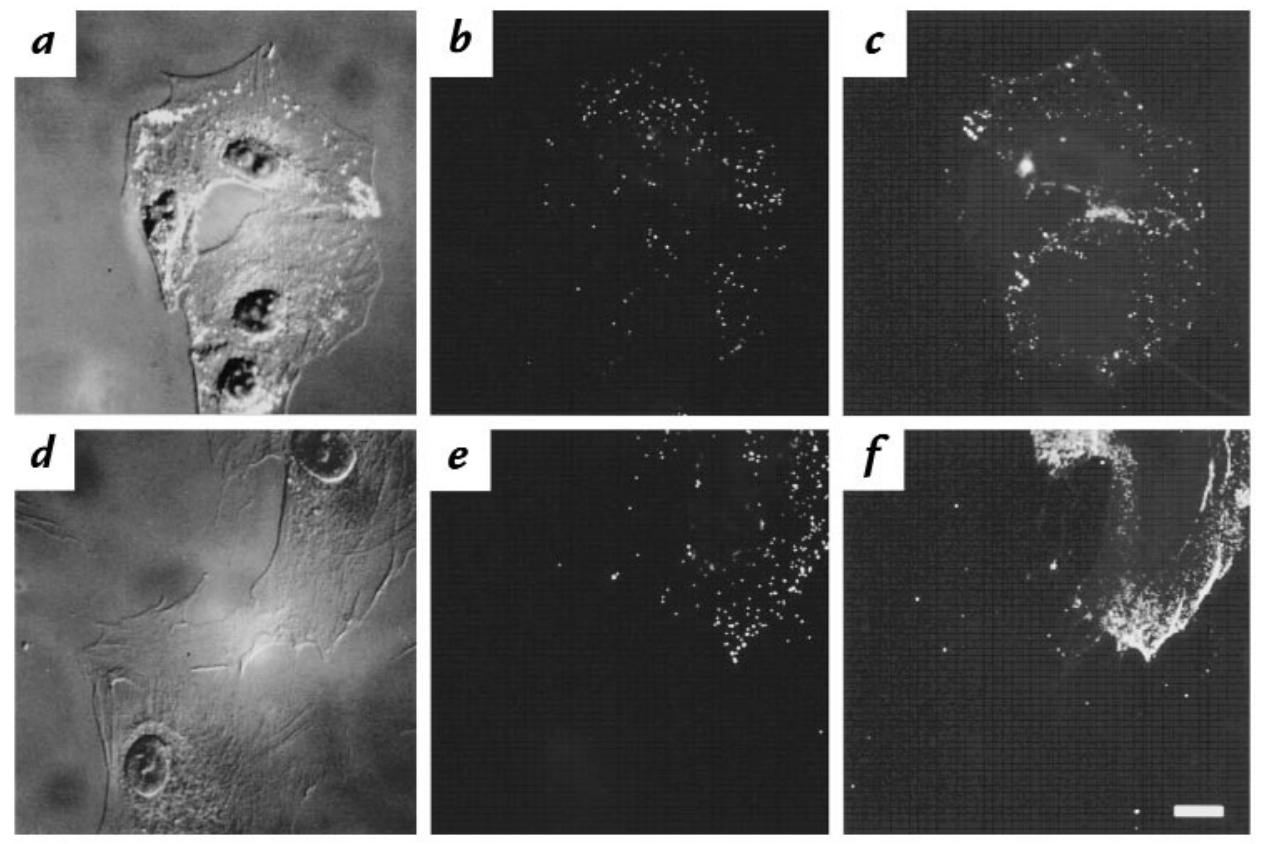

tor, the data also demonstrate that the fiber-CAR interaction is sufficient for Ad entry and expression, although far less efficient than if the RGD-integrin interaction were available. Finally, by altering the Ad fiber to enable attachment to receptors other than CAR, it is possible to use Ad vectors effectively to transfer genes to CAR-deficient, $\alpha_{V}$ integrin-positive cells.

Enhancement of Ad-mediated gene transfer in CAR-sufficient fibroblasts. Previous studies have demonstrated that CAR functions as the high-affinity cell-surface receptor for subgroup C Ad $(8,9)$. Chinese hamster ovary cells $(\mathrm{CHO})$ permanently transfected with CAR are able to bind subgroup C Ad but not subgroup B Ad3 (9) and are able to express genes transferred with an Ad5 vector at least 100fold more efficiently than nontransfected CHO cells (8). Roelvink et al. (31) have demonstrated that Ad subgroups A, C, D, E, and F, but not B, compete for CAR binding. The present study demonstrates that CAR is rate limiting for Ad infection of fibroblasts, and importantly, it

\section{Figure 5}

Correlation of binding of fluorescent Ad vectors with CAR expression. Fibroblasts were infected and labeled with RmcB and Cy3-AdGFP as described in Fig. 4. Fields were selected in AdCAR-infected cultures that included intensely and minimally CAR-positive (green fluorescent) cells. Images were collected, and triplicate gray-scale measurements were made in arbitrary regions within the background (no cells) and within each cell in both green and red channels. CAR expression (green fluorescence) and virus binding (red fluorescence) in a total of 38 cells were expressed as a ratio of cell to background fluorescence. Spearman's correlation analysis revealed a significant correlation, with rho $=0.8$ and $P<0.001$. demonstrates that CAR can improve Ad5 binding and gene transfer to normal, primary human cells (39).

Because Ad infection of primary human dermal fibroblasts is not efficient, the extent of CAR expression after infection with AdCAR is not uniform. This variation allowed us to demonstrate that the extent of Ad binding to a given cell correlates positively with the extent of CAR expression on that individual cell. Upregulation of CAR leads not only to increased Ad binding but enhanced Ad transgene expression. Importantly, this enhancement can be eliminated by the presence of excess recombinant Ad5 fiber. Consistent with the concept that CAR expression enables subgroup-specific Ad entry, a chimeric virus that is Ad5-based but bears Ad7a fiber does not infect CAR-sufficient cells any more efficiently than CAR-deficient cells. This is in agreement with previous findings that subgroup C Ad (serotypes 2 and 5) do not compete for the same receptor as subgroup B Ad (serotypes 3 and 7) $(27,31,40-42)$, and with Tomko et al. (9), who

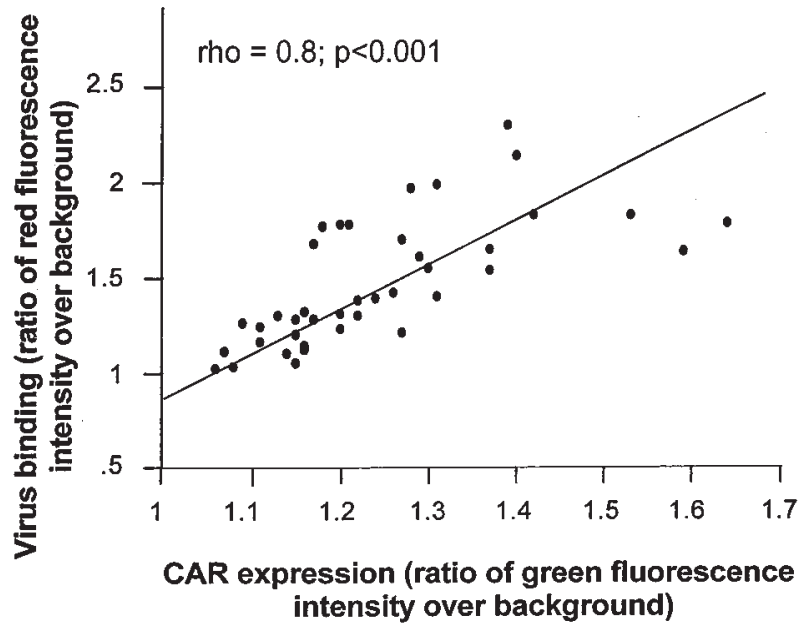




\section{Figure 6}

Enhanced transfer and expression in primary human fibroblasts of the $\beta$ gal transgene by Adßgal after transfer and expression of CAR mediated by AdCAR. Fibroblasts were infected with increasing moi $(0-5 \times$ $10^{4} \mathrm{pu} / \mathrm{cell}$ ) of AdCAR or AdNull. Twenty-four hours later, the cells were infected with $300 \mathrm{pu} / \mathrm{cell}$ of Ad $\beta$ gal. $\beta$ gal activity in cell lysates was assayed $24 \mathrm{~h}$ after the second infection. The dashed line represents the limit of detection of the assay $\left(10^{3}\right.$ relative light units/mg protein). moi, multiplicity of infection. observed that Ad5 bound five times more efficiently than Ad3 to CAR-transfected NIH 3T3 cells.

CAR-independent mechanisms of Ad entry. Whereas upregulation of CAR reflects one strategy for the improvement of Ad-mediated gene transfer to cells, an alternate strategy could be to bypass CAR by altering the fiber of the Ad vector to permit attachment to other receptors. The present study confirms that infection by such fiber-altered vectors do not rely on CAR. On a per particle basis, $\operatorname{AdF}(\mathrm{pK} 7)$ Bgal and AdF(RGD)ßgal were the most efficient at infecting CAR-deficient human fibroblasts, increasing transgene expression by about 100 -fold over that of standard Adßgal. Expression in cells infected by $\operatorname{AdF}(\mathrm{pK} 7) \beta \mathrm{gal}$, which is designed to bind to cells via cell-surface heparan sulfate $(28,29)$, is not affected by the presence or absence of CAR on the cell surface. Although AdF(RGD)Bgal is

\section{Figure 7}

Ability of purified Ad5 fiber to block transfer and expression of the $\beta$ gal gene by Ad $\beta$ gal in CAR-sufficient, but not in CAR-deficient, primary human fibroblasts. Mock-, AdNull-, or AdCAR-infected fibroblasts were incubated with increasing concentrations $(0-1 \mu \mathrm{g} / \mathrm{ml})$ of purified Ad5 fiber at $37^{\circ} \mathrm{C}$ for $1 \mathrm{~h}$ before and during the second infection with $300 \mathrm{pu} / \mathrm{cell}$ of Ad $\beta$ gal. $\beta$ gal activity of cell lysates was assayed $24 \mathrm{~h}$ after the second infection. The data represents mean \pm SE of triplicate measurements.

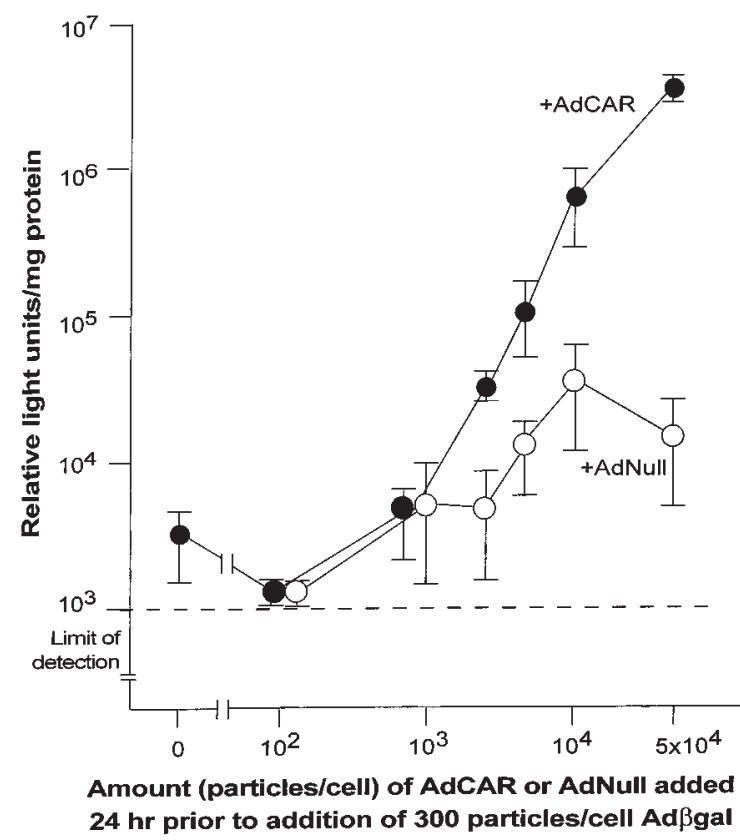

designed to use the RGD- $\alpha_{V}$ integrin interaction for both attachment and internalization (29), the fiber modifications have left the CAR-binding domain available for interaction with CAR. Thus, a modest increase (1.5-fold) in transgene expression in CAR-sufficient vs. CAR-deficient cells is found. The main mechanism of entry, however, is most likely related to the RGD- $\alpha_{V}$ integrin interaction, as the magnitude of the increased transgene expression of this vector over the standard Ad5 vector (70fold) was much greater than that of AdF(RGD) in CARsufficient vs. CAR-deficient cells (1.5-fold).

The lack of significant improvement of infection by AdF9sK $\beta$ gal, an Ad5-based vector with a short fiber and Ad9 knob, in CAR-sufficient versus CAR-deficient fibroblasts was somewhat surprising given that $\mathrm{Ad} 9$ has been demonstrated to compete for binding with $\operatorname{CAR}(30,31)$.

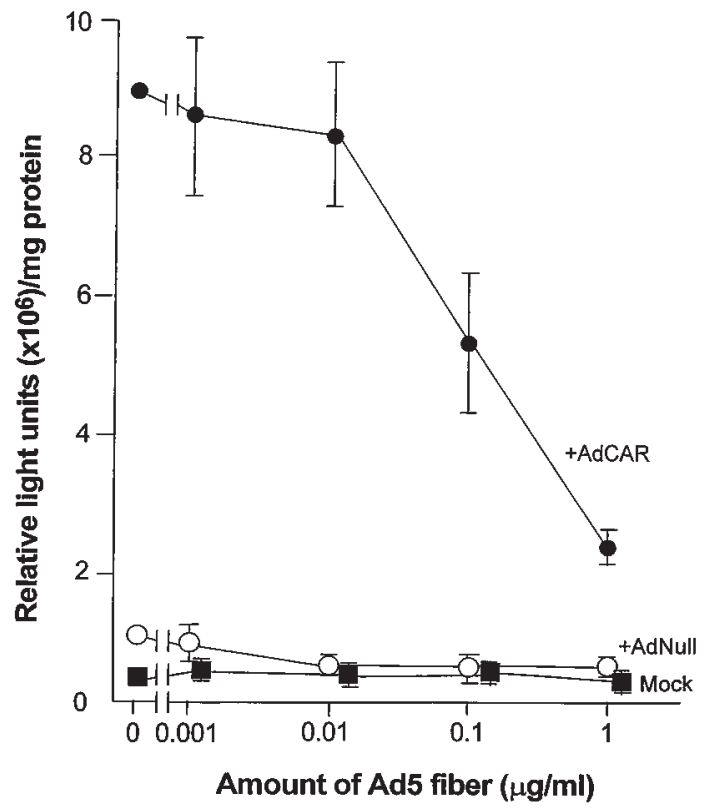


Figure 8

Fiber serotype-specific enhancement of Ad vector-mediated transgene expression in CAR-sufficient versus CAR-deficient cells. Mock-, AdNullor AdCAR-infected fibroblasts were infected with $300 \mathrm{pu} /$ cell of Ad5CAT (Ad5, a standard first-generation Ad5 vector encoding for chloramphenicol acetyl transferase) or Ad5f7CAT (F7, a chimeric Ad5-based vector with the Ad7a fiber encoding for CAT). CAT activity of cell lysates was assayed $24 \mathrm{~h}$ after the second infection. The data represent mean \pm SE of triplicate measurements.

This lack of improvement suggests that the Ad9 knob-CAR interaction is bypassed. The fiber of AdF9sK $\beta$ gal only contains eight of the 22 shaft repeats usually present, and this shortening is designed to enhance entry via the RGD- $\alpha_{v}$ integrin pathway. Expression from this vector in CAR-deficient fibroblasts is only about eightfold greater than that of standard Ad5 vector, whereas $\mathrm{AdF}$ (RGD)ßgal could achieve an approximately 70 -fold greater expression. This observation suggests that, although binding and entry of $\operatorname{AdF}(\mathrm{RGD}) \beta \mathrm{gal}$ and AdF9SK $\beta$ gal may be through the same RGD-mediated mechanism, trafficking of these vectors may not be the same. Alternatively, replacing a subgroup $C$ fiber with a subgroup D fiber could adversely effect particle assembly, resulting in fewer active particles in the vector preparation.

The chimeric vector, Ad5f7CAT, represents another alteration of fiber that enhanced transgene expression relative to standard Ad5 vector in CAR-deficient fibroblasts, albeit to a modest extent (threefold). As the receptor for subgroup B Ad has not been characterized, we cannot determine whether this increase is due to Ad7a fiber binding to its receptor. As with AdF9sKßgal, the Ad7 fiber is somewhat shorter than Ad5 fiber $(30,43)$, and the RGD $-\alpha_{V}$ integrin mechanism may dominate here as well. This may be a more likely explanation, given that Ad7 has not been reported to have a tropism for skin (44).

\section{Figure 9}

Bgal gene transfer and expression mediated by an Ad vector that lacks the penton base RGD domain in CAR-sufficient versus CAR-deficient fibroblasts. Mock-, AdNull-, or AdCAR- infected fibroblasts were infected with $300 \mathrm{pu} / \mathrm{cell}$ of $\mathrm{Ad} \beta \mathrm{gal}(+R G D$, an Ad vector containing a normal penton base RGD sequence) or $A d \triangle R G D \beta g a l$ ( $\triangle R G D$, an Ad vector lacking the penton base RGD sequence). $\beta$ gal activity of cell lysates was assayed 24 $\mathrm{h}$ after the second infection. The dashed line indicates limit of detection of $\beta$ gal assay $\left(10^{3}\right.$ relative light units/mg protein). The data are presented as mean \pm SE of triplicate measurements.

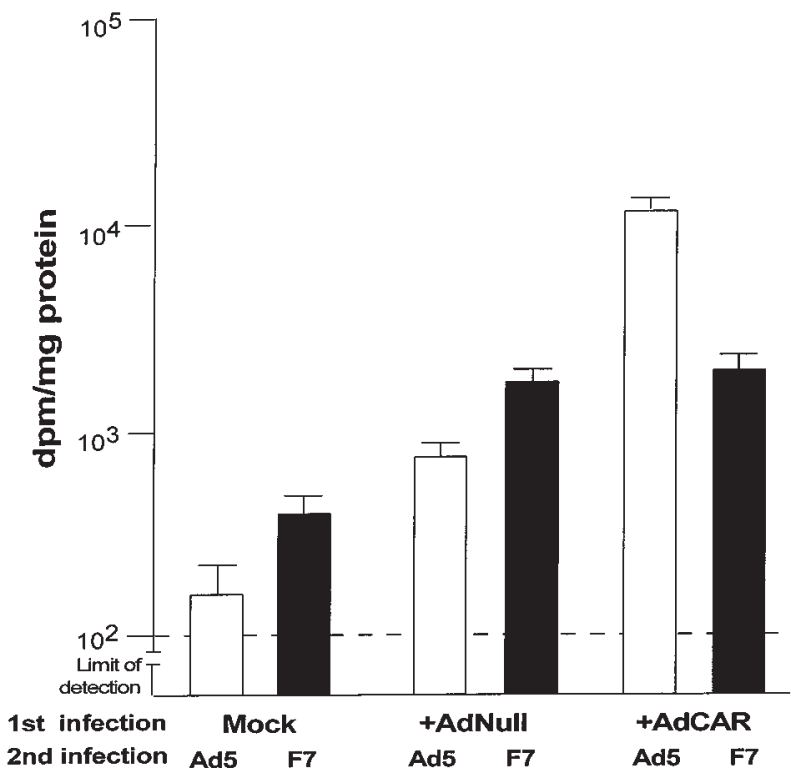

The relative importance of cell-surface $\alpha_{V}$ integrins versus CAR for the entry of Ad into target cells is not clear. The data of Wickham et al. $(4,14)$ demonstrate that Admediated gene transfer is facilitated by an interaction between RGD on the Ad penton base and $\alpha_{V}$ integrin on the cell surface. The present study suggests that even in the absence of RGD- $\alpha_{V}$ integrin interaction, significant gene transfer and expression can be achieved in primary human fibroblasts if adequate fiber-CAR interaction is available, albeit much less (approximately 20-fold) efficiently than in the presence of RGD-integrin interaction. This concept is supported by data demonstrating that RGD-deleted Ad2 can infect fiber receptor-positive cells, but not fiber receptor-negative cells $(7,12)$. The lack of expression by $\mathrm{Ad} \Delta \mathrm{RGD} \beta \mathrm{gal}$ in the naive, CARdeficient cells supports the reciprocal concept that RGD-integrin interaction may be the primary mechanism of CAR-independent gene transfer.

The improved ability of the short fiber vectors over standard Ad5 controls to infect naive fibroblasts also point to the importance of the penton-integrin interaction in CAR-deficient fibroblasts. However, the exclusive use of the penton-integrin entry pathway by the short fiber vectors, including Ad5f7CAT and AdF9sK $\beta$ gal, has not rigorously been proved. All the vectors we have used to demonstrate CAR-independent pathways of entry

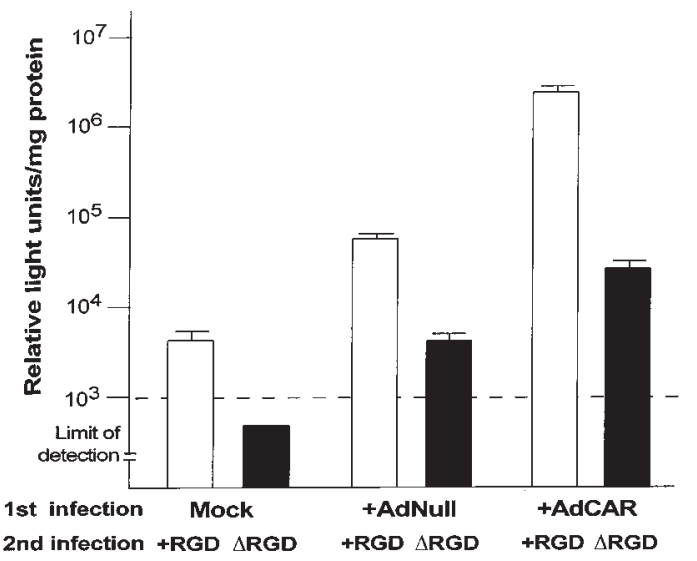




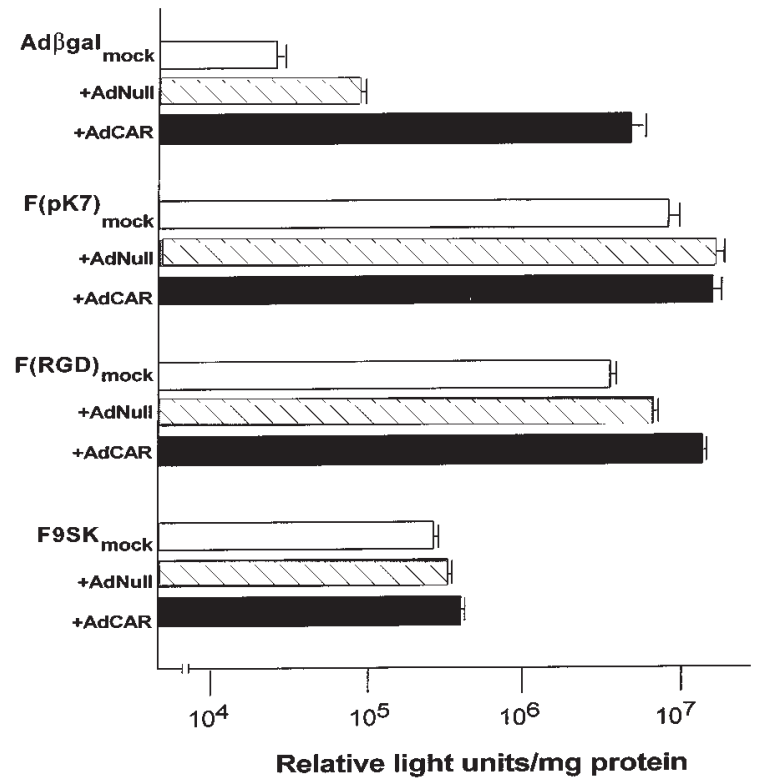

Figure 10

Expression of the transgene in Ad vectors with altered fibers in CAR-sufficient vs. CAR-deficient primary human fibroblasts. Mock-, AdNull-, or AdCAR-infected fibroblasts were infected with $300 \mathrm{pu} / \mathrm{cell}$ of Adßgal (first-generation Ad vector expressing the $\beta$ gal gene) or $\mathrm{AdF}$ (pK7) Bgal $[F(p K 7)$, similar to Adßgal, but seven lysine residues at the end of each fiber protein], AdßgalF(RGD) [F(RGD), similar to Ad $\beta$ gal, but bearing a highaffinity RGD sequence on the end of each fiber protein], or AdF9sK ( $F 9 s$ K, similar to Adßgal, but with a short fiber including the tail and first eight shaft repeats of Ad5 fiber attached to the last shaft repeat and knob of Ad9). Bgal activity of cell lysates was assayed $24 \mathrm{~h}$ after the second infection. The data are presented as mean \pm SE of triplicate measurements.

have normal, RGD-bearing penton bases that probably bind to $\alpha_{V}$ integrin for cell entry. Although the $\mathrm{AdF}(\mathrm{pK} 7)$ Bgal vector is able to infect resting $\mathrm{T}$ cells that are $\alpha_{V}$ integrin-negative $(4,5)$, future studies using fiberaltered vectors that are RGD negative will be necessary to discover whether the penton-integrin interaction is necessary in the absence of fiber-CAR interaction.

The data from our studies suggest that either fiber-CAR interaction or penton-integrin interaction is sufficient to permit Ad entry and gene transfer but that neither is as effective as the two-receptor system used together. Although the fiber-dependent, penton-independent pathway (Ad $\Delta$ RGD $\beta$ gal in CAR-sufficient cells) and the fiber-independent, penton-dependent pathways (short fiber vectors in CAR-deficient cells) led to increases in transgene expression compared with their respective controls, these increases were modest. In contrast, when both mechanisms were available (e.g., standard Ad5 in CAR-sufficient, integrin-positive cells), the increase over that of the control was about 100 -fold. Supporting this hypothesis is the observation that $\operatorname{AdF}(\mathrm{RGD})$ Bgal demonstrates a much higher level of transgene expression than the short fiber viruses, although they all target the $\alpha_{V}$ integrin pathway of entry. Unlike the short fiber vectors, $\mathrm{AdF}(\mathrm{RGD}) \beta \mathrm{gal}$ has both fiber and penton available for binding to cell-surface integrin.

An interesting finding in the control groups was the increase in transgene expression in cells that had been infected with AdNull, an Ad vector with no transgene. The indirect immunofluorescence studies demonstrate that neither $\alpha_{V}$ integrin nor CAR is upregulated by AdNull infection of fibroblasts. That the presence of excess recombinant Ad5 fiber does not affect transgene expression in AdNull-infected cells also suggests that fiber-CAR interaction is not responsible for the increase in transgene expression. The observation of this Null vector effect with every vector tested, including the penton- and fiber-altered Ad vectors, suggests that the Null vector enhancement occurs at a step other than those related to binding or internalization and trafficking. It may be that Ad infection per se activates other biologic processes that, in turn, enhance Ad transgene expression. A recent study by R. Peila et al. (45) suggests that the CMV promoter and, to a lesser extent, the RSV promoter, can be activated by the internalization of Ad. Such activation may be occurring in the present study, because all of the vectors we used included the CMV or RSV promoters. Li et al. (46) have recently described the requirement of PI3-OH kinase activation for Ad internalization. Activation of downstream effectors and other related kinases, such as FAK and MAPK (including ERK1/ERK2), observed early during Ad internalization, may be able to activate the CMV promoter (46). Alternatively, the CMV promoter may be activated by Ad E4 products. Infection with E4-positive vectors has recently been demonstrated to be able to reactivate expression of transgene under either the CMV or RSV promoter that had declined after initial infection via an E4-negative Ad vector in immunodeficient mice (47). In the present study, only E4-positive vectors were used; thus, there could have been enhancement of the CMV-driven transgene by E4-products.

\section{Acknowledgments}

We thank B.G. Harvey of our laboratory for the dermal fibroblasts, M. Harper (Rockefeller University, New York, New York, USA) for helpful discussions regarding statistical analyses, and N. Mohamed for help in preparing the manuscript. These studies were supported in part by the National Institutes of Health (NIH)/National Heart, Lung and Blood Institute (P01 5-P01HL51746, 1-P01-HL59312, and HL51746-03), the Rockefeller University (GCRC NIH M01RR00102), the Cystic Fibrosis Foundation, Will Rogers Memorial Fund, and GenVec Inc. C. Hidaka is supported in part by the Institute for Sports Medicine Research. J. Bergelson is supported by NIH IR02 AI35667 and an Established Investigator Award from the American Heart Association. P. Leopold is supported in part by NIH R29 AI42250.

1. Crystal, R.G. 1995. Transfer of genes to humans: early lessons and obstacles to success. Science. 270:404-410.

2. Shenk, T. 1996. Adenoviridae: the viruses and their replication. In Fields virology. B.N. Fields, D.M. Knipe, and P.M. Howley, editors. LippincottRaven Publishers. Philadelphia, PA. 2111-2148.

3. Wilson, J.M. 1996. Adenoviruses as gene-delivery vehicles. N. Engl. J. Med. 334:1185-1187.

4. Wickham, T.J., Mathias, P., Cheresh, D.A., and Nemerow, G.R. 1993. Integrins $\alpha_{v} \beta_{3}$ and $\alpha_{v} \beta_{5}$ promote adenovirus internalization but not virus attachment. Cell. 73:309-319.

5. Wickham, T.J., Carrion, M.E., and Kovesdi, I. 1995. Targeting of adenovirus penton base to new receptors through replacement of its RGD motif with other receptor-specific peptide motifs. Gene Ther. 2:750-756.

6. Freimuth, P. 1996. A human cell line selected for resistance to adenovirus infection has reduced levels of the virus receptor. J. Virol. 70:4081-4085.

7. Zabner, J., Freimuth, P., Puga, A., Fabrega, A., and Welsh, M.J. 1997. Lack of high affinity fiber receptor activity explains the resistance of 
ciliated airway epithelia to adenovirus infection. J. Clin. Invest. 100:1144-1149.

8. Bergelson, J.M., et al. 1997. Isolation of a common receptor for coxsackie B viruses and adenoviruses 2 and 5. Science. 275:1320-1323.

9. Tomko, R.P., Xu, R., and Philipson, L. 1997. HCAR and MCAR: the human and mouse cellular receptors for subgroup $C$ adenoviruses and group B coxsackieviruses. Proc. Natl. Acad. Sci. USA. 94:3352-3356.

10. Bergelson, J.M., et al. 1998. The murine CAR homolog is a receptor for coxsackie B viruses and adenoviruses. J. Virol. 72:415-419.

11. Hong, S.S., Karayan, L., Tournier, J., Curiel, D.T., and Boulanger, P.A 1997. Adenovirus type 5 fiber knob binds to MHC class I $\alpha 2$ domain at the surface of human epithelial and B lymphoblastoid cells. EMBO J. 16:2294-2306.

12. Bai, M., Harfe, B., and Freimuth, P. 1993. Mutations that alter an ARGGLY-ASP (RGD) sequence in the adenovirus type 2 penton base protein abolish its cell-rounding activity and delay virus reproduction in flat cells. J. Virol. 67:5198-5205.

13. Mathias, P., Wickham, T., Moore, M., and Nemerow, G. 1994. Multiple adenovirus serotypes use $\alpha_{V}$ integrins for infection. J. Virol. 68:6811-6814.

14. Wickham, T.J., Filardo, E.J., Cheresh, D.A., and Nemerow, G.R. 1994. Integrin $\alpha_{V} \beta_{5}$ selectively promotes adenovirus mediated cell membrane permeabilization. J. Cell Biol. 127:257-264.

15. Hashimoto, Y., et al. 1997. Efficient transfer of genes into senescent cells by adenovirus vectors via highly expressed $\alpha_{V} \beta_{5}$ integrin. Biochem. Biophys. Res. Commun. 240:88-92.

16. Takayama, K., et al. 1998 . The levels of integrin $\alpha_{V} \beta_{5}$ may predict the susceptibility to adenovirus-mediated gene transfer in human lung cancer cells. Gene Ther. 5:361-368.

17. Seth, P., Willingham, M.C., and Pastan, I. 1984. Adenovirus-dependent release of ${ }^{51} \mathrm{Cr}$ from $\mathrm{KB}$ cells at an acidic $\mathrm{pH}$. J. Biol. Chem. 259:14350-14353.

18. Seth, P. 1994. Mechanism of adenovirus-mediated endosome lysis: role of the intact adenovirus capsid structure. Biochem. Biophys. Res. Commun. 205:1318-1324.

19. Seth, P. 1994. Adenovirus-dependent release of choline from plasma membrane vesicles at an acidic $\mathrm{pH}$ is mediated by the penton base protein. J. Virol. 68:1204-1206.

20. Panetti, T.S., Wilcox, S.A., Horzempa, C., and McKeown-Longo, P.J. 1995. $\alpha_{V} \beta_{5}$ integrin receptor-mediated endocytosis of vitronectin is protein kinase C-dependent. J. Biol. Chem. 270:18593-18597.

21. Gailit, J. and Clark, R.A. 1996. Studies in vitro on the role of $\alpha_{V}$ and $\beta_{1}$ integrins in the adhesion of human dermal fibroblasts to provisional matrix proteins fibronectin, vitronectin, and fibrinogen. J. Invest. Dermatol. 106:102-108.

22. Gailit, J., et al. 1997. Human fibroblasts bind directly to fibrinogen at RGD sites through integrin $\alpha_{v} \beta_{3}$. Exp. Cell Res. 232:118-126.

23. Mastrangeli, A., et al. 1993. Diversity of airway epithelial cell targets for in vivo recombinant adenovirus-mediated gene transfer. J. Clin. Invest. 91:225-234.

24. Hersh, J., Crystal, R.G., and Bewig, B. 1995. Modulation of gene expression after replication-deficient, recombinant adenovirus-mediated gene transfer by the product of a second adenovirus vector. Gene Ther 2:124-131.

25. Zolotukhin, S., Potter, M., Hauswirth, W.W., Guy, J., and Muzyczka, N. 1996. A "humanized" green fluorescent protein cDNA adapted for highlevel expression in mammalian cells. J. Virol. 70:4646-4654.

26. Frey, B.M., et al. 1998. High-efficiency gene transfer into ex vivo expanded human hematopoietic progenitors and precursor cells by adenovirus vectors. Blood. 91:2781-2792.

27. Gall, J., Kass-Eisler, A., Leinwand, L., and Falck-Pedersen, E. 1996. Adenovirus type 5 and 7 capsid chimera: fiber replacement alters receptor tropism without affecting primary immune neutralization epitopes. J. Virol.
70:2116-2123.

28. Wickham, T.J., Roelvink, P.W., Brough, D.E., and Kovesdi, I. 1996. Adenovirus targeted to heparan-containing receptors increases its gene delivery efficiency to multiple cell types. Nat. Biotech. 14:1570-1573.

29. Wickham, T.J., et al. 1997. Increased in vitro and in vivo gene transfer by adenovirus vectors containing chimeric fiber proteins. J. Virol. 71:8221-8229.

30. Roelvink, P.W., Kovesdi, I., and Wickham, T.J. 1996. Comparative analysis of adenovirus fiber-cell interaction: adenovirus type 2 (Ad2) and Ad9 utilize the same cellular fiber receptor but use different binding strategies for attachment. J. Virol. 70:7614-7621.

31. Roelvink, P.W., Kovesdi, I., Bergelson, J.M., Finberg, R.W., and Wickham, T.J. 1998. Adenovirus retargeting through fiber modification and bifunctional fiber receptor protein (CAR) chimeras. American Society of Gene Therapy, 1st Annual Meeting, Seattle, Washington, May 28-31, 1998; Program and Abstracts.

32. Leopold, P.L., et al. 1998. Fluorescent virions: dynamic tracking of the pathway of adenoviral gene transfer vectors in living cells. Hum. Gene Ther. 9:367-378.

33. Rosenfeld, M.A., et al. 1991. Adenovirus-mediated transfer of a recombinant alpha 1-antitrypsin gene to the lung epithelium in vivo. Science. 252:431-434.

34. Rosenfeld, M.A., et al. 1992. In vivo transfer of the human cystic fibrosis transmembrane conductance regulator gene to the airway epithelium. Cell. 68:143-155.

35. Mittereder, N., March, K.L., and Trapnell, B.C. 1996. Evaluation of the concentration and bioactivity of adenovirus vectors for gene therapy. $J$. Virol. 70:7498-7509.

36. Neumann, J.R., Morency, C.A., and Russian, K.O. 1987. A novel rapid assay for chloramphenicol acetyltransferase gene expression. Biotechniques. 5:444-447.

37. Hsu, K.H., Lonberg-Holm, K., Alstein, B., and Crowell, R.L. 1988. A monoclonal antibody specific for the cellular receptor for the group B $\Delta$ viruses. J. Virol. 62:1647-1652.

38. Pfister, K.K., Wagner, M.C., Stenoien, D.L., Brady, S.T., and Bloom, G.S. 1989. Monoclonal antibodies to kinesin heavy and light chains stain vesicle-like structures, but not microtubules, in cultured cells. J. Cell Biol. 108:1453-1463

39. Leon, R.P., et al. 1998. Adenoviral-mediated gene transfer to lymphocytes. Proc. Natl. Acad. Sci. USA 95:13159-13164.

40. Abrahamsen, K., et al. 1997. Construction of an adenovirus type 7a E1avector. J. Virol. 71:8946-8951.

41. Defer, C., Belin, M.T., Caillet-Boudin, M.L., and Boulanger, P. 1990. Human adenovirus-host cell interactions: Comparative study with members of subgroups B and C. J. Virol. 64:3661-3673.

42. Stevenson, S.C., Rollence, M., White, B., Weaver, L., and McClelland, A. 1995. Human adenovirus serotypes 3 and 5 bind to two different cellular receptors via the fiber head domain. J. Virol. 69:2850-2857.

43. Hong, J.S., Mullis, K.G., and Engler, J.A. 1988. Characterization of the early region 3 and fiber genes of Ad7. Virology . 167:545-553.

44. Horwitz, M.S. 1996. Adenoviruses. In Fields virology. B.N. Fields, D.M. Knipe, and P.M. Howley, editors. Lippincott-Raven Publishers. Philadelphia, PA. 2149-2197.

45. Peila, R., et al. 1998. Adenovirus particle internalization upregulates transgene expression from the CMV promoter. American Society of Gene Therapy, 1st Annual Meeting, Seattle, Washington, May28-31, 1998; Program and Abstracts. 49a. (Abstr.)

46. Li, E., Stupack, D., Klemke, R., Cheresh, D.A., and Nemerow, G.R. 1998. Adenovirus endocytosis via alpha(v) integrins requires phosphoinositide-3-OH kinase. J. Virol. 72:2055-2061.

47. Brough, D.E., et al. 1997. Activation of transgene expression by early region 4 is responsible for a high level of persistent transgene expression from adenovirus vectors in vivo. J. Virol. 71:9206-9213. 